\title{
Hipertensión pulmonar en niños con anemia de células falciformes que viven a gran altura
}

\author{
Ángel Castro Dáger, Adriana Linares Ballesteros
}

Universidad Nacional de Colombia, Fundación HOMI.

Introducción. La anemia de células falciformes (ACF) es una hemoglobinopatía muy frecuente. Su espectro de manifestaciones clínicas es amplio y va desde personas que sufren de enfermedad grave hasta pacientes que tienen manifestaciones clínicas leves. La hipertensión pulmonar (HTP) es una complicación que se ha correlacionado como factor predictor de mayor morbilidad y mortalidad.

Objetivo. Describir y estimar la prevalencia de hipertensión pulmonar en un grupo de pacientes con anemia de células falciformes de la ciudad de Bogotá pertenecientes a la consulta de oncohematología pediátrica.

Material y métodos. Estudio de cohorte transversal. Población blanco: niños menores de 18 años con diagnóstico de anemia de células falciformes por electroforesis de hemoglobina que viven en Bogotá en 2008. Se les realizó ecocardiograma, medición de BNP y marcadores de hemólisis.

Resultados. Quince pacientes, 11 varones (73,3\%), el patrón de electroforesis fue SS en el 66,7\%, S $\beta$ en el $20 \%$ y SC en el $13,3 \%$. Se encontró una edad promedio de 10,7 años, con un porcentaje de HTP del $26,7 \%$, hallándose una tendencia de mayor número de crisis de hiperhemólisis en los niños con hipertensión pulmonar.

Conclusiones. Los niños con ACF que viven a gran altura tienen una prevalencia importante de HTP que inicia a edad más temprana y las crisis de hiperhemólisis se asocian a esta complicación. No se encontró relación entre el BNP y la hipertensión pulmonar. Se debe ampliar la muestra para definir otras correlaciones.

\section{Tumores sólidos en niños en un centro de referencia}

\section{F. Restrepo', A. Linares², I. Sarmiento33, E. Beltrán², S. Castaño², M. Cortés³ L. Jaramillo4, S. Murcia4, M. Méndez} E. Bonilla ${ }^{5}$, F. Fierro ${ }^{5}$, M. P. Aristizábal' ${ }^{5}$ E. Cabrera ${ }^{3}$, F. Ortiz ${ }^{3}$

1 Radioterapeuta, Universidad Nacional de Colombia.

2 Oncohematólogo pediatra, Fundación Hospital de la Misericordia, Universidad Nacional.

${ }^{3}$ Oncohematólogo pediatra, Fundación Hospital de la Misericordia.

${ }^{4}$ Patóloga, Fundación Hospital de la Misericordia, Universidad Nacional.

${ }^{5}$ Cirujano pediatra, Fundación Hospital de la Misericordia, Universidad Nacional.

${ }^{6}$ Oncohematóloga pediatra, Rady Children's Hospital, San Diego (California).

Introducción. La epidemiología del cáncer infantil es variable entre las regiones.

Objetivos. Conocer la frecuencia de los tumores sólidos en un centro de referencia para la atención integral de niños con cáncer.

Material y métodos. Revisión de los diagnósticos en el servicio de patología y oncología de la Fundación Hospital de la Misericordia entre los años 1988 y 2011.

Resultados. Se encontraron 1.902 casos con diagnósticos de tumores malignos sólidos, la causa más frecuente fueron los linfomas: 479; de ellos, 220 casos de linfoma de Hodgkin y 259 linfomas no Hodgkin, seguidos por 447 tumores de SNC, 175 tumores renales, 149 tumores óseos, 126 neuroblastomas, 120 tumores gonadales, 101 histiocitosis, 63 tumores hepáticos, 46 tumores neuroectodérmicos, 42 tumores germinales extragonadales, 33 tumores de glándulas endocrinas, 37 tumores mesenquimales, 13 tumores de glándulas exocrinas y 71 de otros.

Conclusiones. La frecuencia de cáncer es similar a otros centros de referencia de oncología pediátrica en el mundo y un poco diferente al centro de referencia nacional. 